\title{
Three-dimensional fan magnetic reconnection and particle acceleration in the solar corona
}

\author{
Y. E. Litvinenko
}

\author{
Institute for the Study of Earth, Oceans, and Space, University of New Hampshire, Durham, NH 03824-3525, USA \\ e-mail: yuri.litvinenko@unh.edu
}

Received 7 October 2005 / Accepted 22 February 2006

ABSTRACT

\begin{abstract}
Aims. Particle acceleration by the reconnection electric field in three-dimensional magnetic geometries in the solar corona is discussed. The acceleration times, defined by the particle escape from the vicinity of a magnetic null, and the corresponding energy gains are calculated.

Methods. An exact global magnetohydrodynamic solution for fan magnetic reconnection is used to constrain the magnetic and electric fields in the vicinity of the null. Expressions for the particle acceleration times and energy gains are derived by applying the WKB approximation to the equation of motion in nonrelativistic and ultrarelativistic limits.

Results. It is shown that the energies of the accelerated particles can be limited by the particle escape from the null rather than by the total electric potential at the reconnection site. For typical coronal parameters, the finite escape time limits proton energies if the Lundquist number is less than $10^{12}$ and electron energies if the Lundquist number is less than $10^{18}$.

Conclusions. Particle acceleration by the electric field, associated with fan magnetic reconnection in solar flares, can explain proton energies of the order of a few $\mathrm{MeV}$ and electron energies of the order of a few hundred $\mathrm{keV}$ in the case of classical electric resistivity. Energies up to a few hundred $\mathrm{MeV}$ can be reached if the resistivity at the reconnection site is turbulent. These estimates agree with typical solar flare observations.
\end{abstract}

Key words. acceleration of particles - Sun: flares - Sun: particle emission

\section{Introduction}

Electrons with energies above a few $\mathrm{keV}$ and protons with energies above a few hundred $\mathrm{keV}$ are responsible for the observed solar flare radiation in a broad energy range. These nonthermal particles can contain a large fraction of the total flare energy. Recent measurements with instruments onboard the RHESSI spacecraft imply that electrons with energies above $20 \mathrm{keV}$ can contain up to half of the total flare energy, at least in smaller impulsive flares (Lin et al. 2003). Although a complete theoretical understanding of particle acceleration in flares is still a long way off, magnetic reconnection provides a single physical cause for the overall energy release and particle acceleration (Priest $\&$ Forbes 2000). Three-dimensional reconnection experiments confirm that strong electric fields, associated with rapid reconnection, can accelerate charged particles well above the thermal energies (e.g., Brown et al. 2002).

Multiwavelength observations of the solar corona strongly suggest that three-dimensional magnetic reconnection is the key mechanism of energy release in solar flares (Aulanier et al. 2000; Fletcher et al. 2001). These observations are detailed enough to identify the "spine" curves and "fan" surfaces that define the structure of three-dimensional magnetic nulls (Priest \& Titov 1996). Physically, spine-type reconnection is caused by plasma motion across the fan surface, which generates a localized electric current along the spine. Fan-type reconnection, on the other hand, is caused by motion across the spine, which generates a global current sheet aligned with the fan. Exact analytical magnetohydrodynamic (MHD) solutions have been discovered, which describe both spine and fan magnetic reconnection (Craig et al. 1995; Craig \& Fabling 1996; Heerikhuisen \& Craig 2005).
When the number of high-energy particles, accelerated at a reconnection site, is small enough, it is useful to consider the motion of a test particle. In this approximation, particle interaction with other particles and the changes in the imposed electric and magnetic fields caused by the acceleration process are neglected. Test particle orbits in two-dimensional reconnection geometries (an X-point or a current sheet) have been obtained both analytically and numerically (Speiser 1965; Bulanov \& Sasorov 1976; Martens 1988; Martens \& Young 1990). The effects of a three-dimensional magnetic field with a component parallel to the electric field have also been explored (Bruhwiler \& Zweibel 1992; Litvinenko 1996; Browning \& Vekstein 2001; Litvinenko 2003; Wood \& Neukirch 2005; Efthymiopoulos et al. 2005). A weakness of these studies, however, is that they adopted simplified magnetic and electric fields that reproduced the basic reconnection topology qualitatively but did not follow from a quantitative reconnection solution.

A physically motivated technique for investigating test particle orbits in two-dimensional current sheets (Heerikhuisen et al. 2002; Craig \& Litvinenko 2002) is based on the use of analytical global MHD solutions for magnetic reconnection. In this technique the properties of the reconnection site are not free parameters but rather follow from the reconnection solutions (Craig \& Henton 1995; Craig et al. 1995), leading to quantitative predictions for the properties of energetic particle distributions.

Particles have also been traced in turbulent magnetic and electric fields, computed with various resistive MHD codes (Ambrosiano et al. 1988; Schopper et al. 1999; Dmitruk et al. 2003; Turkmani et al. 2005), but limited numerical resolution makes it difficult to interpret the results and scale them to the parameters of the solar corona. 
Few studies so far have addressed the question of particle acceleration at a single three-dimensional reconnection site. Bulanov \& Cap (1988) developed an analytical technique for obtaining WKB solutions for particle trajectories near a general magnetic null and presented a detailed calculation for the case of an almost one-dimensional current sheet. Dalla \& Browning (2005) computed particle orbits near a potential magnetic null with the electric field given by a linearized ideal MHD solution for slow spine reconnection (Priest \& Titov 1996).

The aim of this paper is to calculate the energy gains of particles, which result from acceleration by the reconnection electric field in the fan plane of a magnetic null. The analytical technique suggested by Bulanov \& Cap (1988) is combined with the fan reconnection solution derived by Craig \& Fabling (1996) and the resulting estimates for particle energy gains are applied to the problem of particle acceleration by magnetic reconnection in solar flares. The analysis is focused on fan reconnection rather than spine reconnection because fan reconnection can provide much larger energy release rates in the solar corona (Craig \& Watson 2000).

This study of particle acceleration by three-dimensional magnetic reconnection extends the analysis of a similar problem in two-dimensional X-point and separator geometries (Heerikhuisen et al. 2002; Craig \& Litvinenko 2002). Note for clarity that the approach does not allow one to calculate the flux of accelerated particles, which would be necessary in order to address "the number problem" for flare-accelerated particles (e.g., Lin et al. 2003).

\section{Fan magnetic reconnection}

Exact solutions for three-dimensional magnetic reconnection can be constructed in the framework of incompressible MHD by assuming that both the magnetic field and the plasma velocity are composed of a background potential component and a reconnection-related component (Craig \& Fabling 1996). The simplest background field is a linear null point. In the case when there is no rotation of the magnetic field vector as it is advected along the spine, the steady fan reconnection solution for the magnetic field is given by

$\boldsymbol{B}=\left[\frac{\lambda}{2} x+X(z), \frac{\lambda}{2} y,-\lambda z\right]$

(see Craig \& Fabling 1996, for derivation and the expression for $X)$. The solution contains a current sheet in the fan plane $z=0$. The electric current density, $\boldsymbol{J}=(c / 4 \pi) \nabla \times \boldsymbol{B} \sim X^{\prime}(z)$, is localized on the scale of the current sheet thickness $l$. The electric field is defined by Ohm's law:

$\boldsymbol{E}=\eta \boldsymbol{J}-\frac{\boldsymbol{u}}{c} \times \boldsymbol{B}$,

where $\eta$ is the electric resistivity and $\boldsymbol{u}$ is the plasma velocity.

This MHD solution contains several parameters that need to be specified in some physically appropriate manner. One possibility is the so-called saturated flux pile-up reconnection regime (Litvinenko \& Craig 2000). Because the reconnecting magnetic field at the entrance to the current sheet cannot be arbitrarily large, a finite field is specified at the edge of the sheet, $B_{\mathrm{s}} \simeq X(z=l)$. Additionally, the reconnection outflow is limited by the local Alfvén speed. These conditions lead to

$\lambda \simeq \frac{B_{\mathrm{s}}}{L_{\mathrm{c}}}$

$$
l \simeq \sqrt{\frac{c^{2} \eta L_{\mathrm{c}}}{4 \pi v_{\mathrm{A}}} \frac{B_{\mathrm{c}}}{B_{\mathrm{s}}}}
$$

(Craig \& Watson 2000). Here typical values of the Alfvén speed $v_{\mathrm{A}}$, magnetic field $B_{\mathrm{c}}$, and length scale $L_{\mathrm{c}}$ (say, in the solar corona) are used. The global solution is complete once the resistivity $\eta$ and the magnetic field $B_{\mathrm{s}}$ are specified.

An advantage of the fan reconnection solution is that it can provide flare-like energy release rates. The maximum rate for the fan solution scales as $\sim \eta^{1 / 2} B_{\mathrm{s}}^{5 / 2}$ and can reach $10^{28} \mathrm{erg} \mathrm{s}^{-1}$ for reasonable coronal parameters (Craig \& Watson 2000). In contrast, the scaling for the spine solution, $\sim \eta B_{\mathrm{s}}^{2}$, is much less favorable, given the large Lundquist number in the solar corona. Physically, electric currents in the spine reconnection solution are too localized to account for the flare energy output.

Finally, consider the expressions for the electric and magnetic fields near the fan plane. The electric field is

$\boldsymbol{E} \simeq[0, E, 0]$,

and the reconnecting magnetic field is

$X(z) \simeq h z$.

The local field gradient is related to the electric field:

$h=\frac{B_{\mathrm{s}}}{l}=\frac{4 \pi E}{c \eta}$.

\section{Particle orbits in three-dimensional magnetic nulls}

The reconnection electric field accelerates charged particles if there is an electric field component along the magnetic field. In the case of fan magnetic reconnection, Eqs. (1) and (2) show that $\boldsymbol{E} \cdot \boldsymbol{B} \sim B_{y} X^{\prime}$. Because $X^{\prime}$ rapidly falls off away from the fan plane $z=0$, the fan is the site of the most efficient particle acceleration.

The energy gain for a particle of charge $e$ is given by the electric potential difference, which is defined by the product of the field magnitude $E$ and the acceleration length $\leq L_{\mathrm{c}}$. The maximum energy gain would be

$\mathcal{E}_{\max }=e E L_{\mathrm{c}}$

if the magnetic field in the fan could be ignored. The magnetic field near the null, however, generally results in a Lorentz force component that ejects the particle away from the fan. Once the particle exits the nonadiabatic region near the fan, further direct electric field acceleration is impossible since the electric field outside the current sheet has no component along the magnetic field. This is why the final kinetic energy (neglecting betatron acceleration in the drift region) is defined by the ejection time, which is a function of the electric and magnetic fields in the vicinity of the null.

Bulanov \& Cap (1988) suggested an analytical technique in order to describe the process of particle acceleration at threedimensional magnetic nulls. Below, this general technique is applied to the particular geometry of fan magnetic reconnection. Because efficient particle acceleration is only possible in the vicinity of the fan, it is sufficient to use the simple expressions for the electric and magnetic fields, given at the end of Sect. 2. It should be emphasized that $\lambda, h$ and $E$ are not independent parameters but rather are determined by the global reconnection solution of Craig \& Fabling (1996). For generality and ease of 
comparison with other models, though, the analysis of this section is carried out as if the parameters were independent.

Test particle orbits are determined by solving the equation of motion

$\dot{\boldsymbol{p}}=e\left(\boldsymbol{E}+\frac{1}{c} \boldsymbol{v} \times \boldsymbol{B}\right)$,

where $\boldsymbol{p}=\gamma m \boldsymbol{v}, \boldsymbol{v}, e, m$ are the momentum, velocity, charge and mass of the particle, and $\gamma$ is the relativistic Lorentz factor.

\subsection{Nonrelativistic limit}

The nonrelativistic equation of motion in coordinate form is as follows:

$\ddot{x}=\frac{e \lambda}{m c}\left(-\dot{y} z-\frac{1}{2} y \dot{z}\right)$,

$\ddot{y}=\frac{e E}{m}+\frac{e}{m c}\left(h z \dot{z}+\frac{1}{2} \lambda x \dot{z}+\lambda \dot{x} z\right)$,

$\ddot{z}=\frac{e}{m c}\left(\frac{1}{2} \lambda \dot{x} y-\frac{1}{2} \lambda \dot{x} \dot{y}-h \dot{y} z\right)$,

where Eqs. (1), (5), and (6) have been used.

The reconnecting magnetic field component generally acts to prevent the particle escape from the current sheet (Speiser 1965). This can be shown for fan reconnection by setting $\lambda=0$, integrating the second equation, and substituting the resulting $\dot{y}(t)$ into the last equation:

$\ddot{z}=-\frac{e h}{m c}\left(\frac{e E t}{m}+\frac{e h}{2 m c}\left(z^{2}-z_{0}^{2}\right)\right) z$,

where $z_{0}$ is the initial position. Here and in what follows, the initial particle velocity is neglected. Clearly this equation describes stable oscillations with the frequency $\sim t^{1 / 2}$ around the fan plane $z=0$. Thus it is the background field that causes the particle to escape from the fan, and the escape time must depend on the parameter $\lambda$ that characterizes the gradient of the potential magnetic field. The orbit instability corresponds to rapid escape of the particle from the vicinity of the null, which prevents further acceleration by the electric field (Bulanov \& Cap 1988).

Turning to the case $\lambda \neq 0$, note that a particular exact solution is valid for any value of $\lambda$ :

$x=0, \quad y=y_{0}+\frac{e E}{2 m} t^{2}, \quad z=0$.

It describes acceleration along the straight line in the fan where the electric and magnetic fields are coaligned. The solution describes the particles that are initially in the fan plane. The behavior of particles with nonzero initial positions $x_{0}$ and $z_{0}$ can be obtained by perturbing the equation of motion around the exact solution. The procedure is to take the unperturbed solution $y=y(t)$ above and substitute it into Eqs. (10) and (12) that become linear differential equations for the perturbations $x(t)$ and $z(t)$. Bulanov \& Cap (1988) suggested to look for solutions of the two resulting equations in the form $x(t)=$ $\sum_{k=1}^{4} x_{k} \exp \left(\int \omega_{k} \mathrm{~d} t\right)$ and $z(t)=\sum_{k=1}^{4} z_{k} \exp \left(\int \omega_{k} \mathrm{~d} t\right)$, assuming that the temporal variation of $x_{k}$ and $z_{k}$ can be ignored. Bulanov \& Cap (1988) argued that the WKB method is justified because the numerical coefficients in the first-order equations for $x(t)$ and $z(t)$ are large and that the solution is valid in the region where the particles are unmagnetized. The size of the region is of the order of $\left(m c^{2} E / e \lambda^{2}\right)^{1 / 3}$, which is consistent with Eq. (20).

The method leads to the following equation for the growth rates $\omega_{k}$ :

$\omega^{2}\left(\omega^{2}+\frac{e h}{m c} \dot{y}\right)=\frac{1}{2}\left(\frac{e \lambda}{m c}\right)^{2}\left(\dot{y}+\frac{1}{2} \omega y\right)(\dot{y}-\omega y)$.

The motion in the vicinity of the null is unstable for some initial conditions if this equation has positive real solutions for $\omega$. As a check of the method, note that the motion is predicted to be stable for $\lambda=0$, consistent with the exact Eq. (13).

If $\dot{y} \ll \omega y$, all solutions of Eq. (15) are imaginary, and hence the motion is stable. For example, if additionally $\dot{y} \ll \omega^{2} m c / e h$, then the solution is

$\omega(t) \simeq \pm \frac{i}{2} \frac{e \lambda}{m c}|y(t)|$

Now the solution for $\omega(t)$ can be used to show that the requirement $\dot{y} \ll \omega y$ corresponds to a weak electric field. Specifically, the opposite of Eq. (20) has to be satisfied. The other limit $\dot{y} \gg \omega y$ describes efficient particle accelerated at the null by a strong electric field.

If $\dot{y} \gg \omega y$, Eq. (15) simplifies to

$\omega^{2}\left(\omega^{2}+\frac{e h}{m c} \dot{y}\right) \simeq \frac{1}{2}\left(\frac{e \lambda}{m c}\right)^{2} \dot{y}^{2}$.

Given the approximate nature of the method, it does not make sense to consider the exact solution to this equation. Consider instead two limiting cases. If $\dot{y} \ll \omega^{2} m c / e h$, then a positive real solution for $\omega$ is present,

$\omega \simeq\left(\frac{e^{2} \lambda E t}{2^{1 / 2} m^{2} c}\right)^{1 / 2}$,

indicating that the particle is indeed ejected from the vicinity of the null. The acceleration time is defined as $\int_{0}^{t_{\text {acc }}} \omega(t) \mathrm{d} t \simeq 1$ that leads to

$t_{\mathrm{acc}} \simeq\left(\frac{m^{2} c}{e^{2} \lambda E}\right)^{1 / 3}$

ignoring factors of order unity. A more rigorous estimate would take into account the initial position of the particle (Bulanov \& Cap 1988). Now $\omega\left(t_{\text {acc }}\right)$ can be substituted into $\dot{y} \gg \omega y$, which gives

$\frac{m c^{2} E}{e \lambda^{2} y^{3}} \gg 1$

This result confirms that the reconnection electric field must be strong enough and particles must be close enough to the null to justify the approximation $\dot{y} \gg \omega y$. The condition $\dot{y} \ll \omega^{2} m c / e h$ becomes simply $h \ll \lambda$, which means that the reconnectionrelated magnetic field is small compared with the background field.

Alternatively if $\dot{y} \gg \omega y$ as before but $h \gg \lambda$, then Eq. (17) is solved to give an unstable solution characterized by

$\omega \simeq\left(\frac{e^{2} \lambda^{2} E t}{2^{1 / 2} m^{2} c h}\right)^{1 / 2}, \quad t_{\mathrm{acc}} \simeq\left(\frac{m^{2} c h}{e^{2} \lambda^{2} E}\right)^{1 / 3}$.

The requirement $h \gg \lambda$ means that the reconnecting magnetic field has to be strong compared with the background field. 
Thus, as long as the reconnection electric field is strong enough, the acceleration time in the vicinity of a magnetic null is defined by Eq. (19) if $h \ll \lambda$ and Eq. (21) if $h \gg \lambda$. The resulting particle ejection from the null limits the nonrelativistic energy gain to

$\mathcal{E}_{3 \mathrm{~d}} \simeq \frac{e^{2} E^{2}}{2 m} t_{\mathrm{acc}}^{2}$

The actual energy gain is determined by the smaller of $\mathcal{E}_{3 \mathrm{~d}}$ and $\mathcal{E}_{\max }$.

\subsection{Ultrarelativistic limit}

Analogous results can be derived for the particle motion in the relativistic regime. The equation of motion in coordinate form is as follows:

$\dot{p}_{x}=\frac{e \lambda}{c}\left(-\dot{y} z-\frac{1}{2} y \dot{z}\right)$,

$\dot{p}_{y}=e E+\frac{e}{c}\left(h z \dot{z}+\frac{1}{2} \lambda x \dot{z}+\lambda \dot{x} z\right)$,

$\dot{p}_{z}=\frac{e}{c}\left(\frac{1}{2} \lambda \dot{x} y-\frac{1}{2} \lambda x \dot{y}-h \dot{y} z\right)$.

Acceleration along the $y$-axis in the fan plane is described by a particular exact solution

$x=0, \quad p_{y}=e E t, \quad z=0$.

As in the nonrelativistic limit, the acceleration times are determined by deriving a perturbed solution that is valid approximately for general nonzero initial conditions. Assume for simplicity that the energy $\mathcal{E}(t) \simeq c e E t \gg m c^{2}$ (ultrarelativistic limit), implying that $y(t) \simeq y_{0}+c t$ and $\dot{y} \simeq c$. Using the particular exact solution, the perturbed equations for $x(t)$ and $z(t)$ are written as follows:

$\ddot{x}+\frac{1}{t} \dot{x} \simeq-\frac{\lambda}{E t}\left(c z+\frac{1}{2} y \dot{z}\right)$,

$\ddot{z}+\frac{1}{t} \dot{z} \simeq \frac{1}{E t}\left(\frac{1}{2} \lambda \dot{x} y-\frac{1}{2} \lambda x c-h c z\right)$.

Here the derivative of the momentum component $p_{x}$ is conveniently expressed as $\dot{p}_{x}=(\dot{\mathcal{E}} \dot{x}+\mathcal{E} \ddot{x}) / c^{2}$ and similarly for the $z$-component.

The perturbed equations for $x(t)$ and $z(t)$ are solved using the WKB approximation $\sim \exp \left(\int \omega \mathrm{d} t\right)$. The solution of the resulting equation for $\omega$ shows that the motion is stable if $\omega y \gg c$. If $\omega y \ll c$, the growth rates are determined by the following equation:

$\omega^{2}\left(\omega^{2}+\frac{c h}{E t}\right) \simeq \frac{1}{2}\left(\frac{c \lambda}{E t}\right)^{2}$.

Clearly positive growth rates are possible for any values of $\lambda$ and $h>0$ as long as $\lambda \neq 0$. Keeping the same level of accuracy as in the nonrelativistic limit, consider two limiting cases. If $h \ll \lambda$, the unstable solution is given by

$\omega \simeq\left(\frac{c \lambda}{2^{1 / 2} E t}\right)^{1 / 2}, \quad t_{\mathrm{acc}} \simeq \frac{E}{c \lambda}$.
Conversely, if $h \gg \lambda$, the unstable solution is given by

$\omega \simeq\left(\frac{c \lambda^{2}}{2 h E t}\right)^{1 / 2}, \quad t_{\mathrm{acc}} \simeq \frac{h E}{c \lambda^{2}}$.

The energy gain in the ultrarelativistic limit is given by

$\mathcal{E}_{3 \mathrm{~d}} \simeq c e E t_{\mathrm{acc}}$.

As a check of the calculation, note that the results for the ultrarelativistic limit could be reproduced by the substitution $m c^{2} \rightarrow \mathcal{E}_{3 \mathrm{~d}}$ in the nonrelativistic formulae for $\mathcal{E}_{3 \mathrm{~d}}$.

\section{Particle acceleration in fan reconnection models}

The results of Sect. 3, combined with the exact fan reconnection solution of Sect. 2, can be applied to the problem of particle acceleration in solar flares. It is useful to adopt normalizing values for the magnetic field $B_{\mathrm{c}}$, the size of a flaring active region $L_{\mathrm{c}}$, and the Alfvén speed $v_{\mathrm{A}}$. Typical coronal values $B_{\mathrm{c}}=10^{2} \mathrm{G}$, $L_{\mathrm{c}}=10^{9.5} \mathrm{~cm}$, and the plasma density $\rho_{\mathrm{c}}=10^{-15} \mathrm{~g} \mathrm{~cm}^{-3}$ identify $v_{\mathrm{A}}=B_{\mathrm{c}} / \sqrt{4 \pi \rho_{\mathrm{c}}} \simeq 10^{9} \mathrm{~cm} \mathrm{~s}^{-1}$.

In the fan reconnection solution of Sect. 2 , the potential and nonpotential field gradients $(\lambda$ and $h$ ) and the reconnection electric field $E$ are related through Eqs. (3) and (7). Equation (4) gives the dimensionless thickness of the current sheet in the fan:

$\tilde{l} \simeq \tilde{\eta}^{1 / 2} \tilde{B}_{\mathrm{s}}^{-1 / 2}$

where $\tilde{B}_{\mathrm{s}}$ is the dimensionless field at the edge of the sheet. The dimensionless electric resistivity $\tilde{\eta}=c^{2} \eta /\left(4 \pi v_{\mathrm{A}} L_{\mathrm{c}}\right)$ is the inverse of the Lundquist number. Here and below the tilde is used to denote dimensionless quantities. The dimensionless electric current density in the fan, $\tilde{h} \simeq \tilde{B}_{\mathrm{s}} / \tilde{l}$, scales as

$\tilde{h} \simeq \tilde{\eta}^{-1 / 2} \tilde{B}_{\mathrm{s}}^{3 / 2}$,

leading to

$\frac{h}{\lambda} \simeq \tilde{\eta}^{-1 / 2} \tilde{B}_{\mathrm{s}}^{1 / 2}$

Ohm's law $\tilde{E}=\tilde{\eta} \tilde{h}$ gives the dimensionless electric field in the fan, which quantifies the reconnection rate:

$\tilde{E} \simeq \tilde{\eta}^{1 / 2} \tilde{B}_{\mathrm{s}}^{3 / 2}$

The electric field is measured in units of $v_{\mathrm{A}} B_{\mathrm{c}} / c$.

Note that the scaling with resistivity $\tilde{E} \sim \tilde{\eta}^{1 / 2}$ is the same as that for the Sweet-Parker current sheet. The present reconnection model, however, also incorporates an important effect of flux pile-up at the edge of the current sheet. The effect is quantified by the pile-up factor $\tilde{B}_{\mathrm{s}} \geq 1$. Larger pile-ups lead to thinner sheets, faster reconnection rates, stronger electric fields and higher particle energies.

The electric resistivity $\tilde{\eta}$ in the solar corona can be as small as $10^{-14}$ for the classical resistivity due to Coulomb collisions and perhaps as large as $10^{-8}$ for anomalous turbulent resistivity (e.g., LaRosa 1992). Reconnection models based on the classical resistivity formally predict current carrier speeds that approach or even exceed the speed of light $c$. In reality collisional conditions in the current sheet break down and current-driven instabilities limit the electron drift speed to a value on the order of the proton sound speed $\simeq 10^{-3} \mathrm{c}$. In the absence of a generally accepted theory of turbulent magnetic reconnection, $\tilde{\eta}$ is treated as a parameter in the remainder of this paper. 
Consider first the energy gain for nonrelativistic particles. Equation (35) shows that $h / \lambda \gg 1$ because $\tilde{\eta} \ll 1$. Using Eqs. (21), (22), (34)-(36), the energy gain is expressed as follows:

$\frac{\mathcal{E}_{3 \mathrm{~d}}}{m v_{\mathrm{A}}^{2}} \simeq\left(\frac{e B_{\mathrm{c}} L_{\mathrm{c}}}{m c v_{\mathrm{A}}}\right)^{2 / 3} \tilde{\eta}^{1 / 3} \tilde{B}_{\mathrm{s}}^{5 / 3}$.

An important point is that for $\tilde{\eta}$ close to zero this scaling is more favorable than the scaling for the maximum possible energy gain, defined by Eq. (8):

$\frac{\mathcal{E}_{\max }}{m v_{\mathrm{A}}^{2}} \simeq\left(\frac{e B_{\mathrm{c}} L_{\mathrm{c}}}{m c v_{\mathrm{A}}}\right) \tilde{\eta}^{1 / 2} \tilde{B}_{\mathrm{s}}^{3 / 2}$.

The condition $\mathcal{E}_{3 \mathrm{~d}}>\mathcal{E}_{\max }$ identifies the regime of efficient particle acceleration by three-dimensional magnetic reconnection. Physically it means that the ejection from the null by the Lorentz force does not occur rapidly enough. Therefore the particles can be accelerated by the electric field in the fan to energies on the order of $\mathcal{E}_{\text {max }}$.

The requirement $\mathcal{E}_{3 \mathrm{~d}}>\mathcal{E}_{\max }$ yields

$\tilde{\eta}<\left(\frac{m c v_{\mathrm{A}}}{e B_{\mathrm{c}} L_{\mathrm{c}}}\right)^{2} \tilde{B}_{\mathrm{s}}$.

The typical values $L_{\mathrm{c}}, B_{\mathrm{c}}$, and $v_{\mathrm{A}}$ above lead to $\tilde{\eta}<10^{-13} \tilde{B}_{\mathrm{s}}$ for protons. Therefore, for a realistic pile-up factor $1<\tilde{B}_{\mathrm{s}}<10$, the proton energy gain in flares will not be limited by rapid ejection away from the null unless $\tilde{\eta}$ is anomalously enhanced. Yet the corresponding requirement for electrons, $\tilde{\eta}<10^{-19} \tilde{B}_{\mathrm{s}}$, is almost impossible to satisfy. Hence typical electron energies will be less than the maximum value $\mathcal{E}_{\max }$.

In the ultrarelativistic limit, the energy gain is given by Eqs. (31) and (32) in the case $h / \lambda \gg 1$. Using Eqs. (34)-(36) gives

$\mathcal{E}_{3 \mathrm{~d}} \simeq e B_{\mathrm{c}} L_{\mathrm{c}} \frac{v_{\mathrm{A}}^{2}}{c^{2}} \tilde{\eta}^{1 / 2} \tilde{B}_{\mathrm{s}}^{5 / 2}$,

and the requirement $\mathcal{E}_{3 \mathrm{~d}}>\mathcal{E}_{\max }$ results in

$\tilde{B}_{\mathrm{s}}>c / v_{\mathrm{A}} \simeq 30$

for the reference value $v_{\mathrm{A}}=10^{9} \mathrm{~cm} \mathrm{~s}^{-1}$. The condition is difficult to satisfy for realistic pile-up factors $1<\tilde{B}_{\text {s }}<10$. Hence relativistic energies of both protons and electrons will be less than the maximum value $\mathcal{E}_{\max }$.

Is efficient particle acceleration possible at threedimensional reconnection sites in the solar corona? As an example, consider particle acceleration in the fan reconnecting current sheet with the pile-up factor $\tilde{B}_{\mathrm{s}} \simeq 5$, corresponding to the local magnetic field on the order of $500 \mathrm{G}$. If the electric resistivity in the sheet is collisional, $\tilde{\eta} \simeq 10^{-14}$, then the condition $\mathcal{E}_{3 \mathrm{~d}}>\mathcal{E}_{\max }$ is satisfied for nonrelativistic protons, and the protons can reach the energies up to $\mathcal{E}_{\max } \simeq 3 \mathrm{MeV}$. Although most flare-accelerated protons indeed have energies within the range $0.1-10 \mathrm{MeV}$, the highest proton energies observed are in the $1-5 \mathrm{GeV}$ range (Ryan 2000). Stronger electric fields are required to produce the relativistic protons. If rapid magnetic reconnection is associated with anomalous resistivity on the order of $\tilde{\eta} \simeq 10^{-8}$, then the maximum proton energy is formally $\mathcal{E}_{\max } \simeq 3 \mathrm{GeV}$. Because $\mathcal{E}_{3 \mathrm{~d}}<\mathcal{E}_{\max }$, however, particles are ejected from the fan before the maximum energy can be reached. Nevertheless the final energies are on the order of $\mathcal{E}_{3 \mathrm{~d}} \simeq 0.5 \mathrm{GeV}$, which is a promising result, given the approximate nature of the calculation.

Because electrons are rapidly ejected from the fan in both nonrelativistic and ultrarelativistic limits, their energies are determined by the corresponding formulae for $\mathcal{E}_{3 \mathrm{~d}}$ rather than $\mathcal{E}_{\max }$. For the pile-up factor $\tilde{B}_{\mathrm{s}} \simeq 5$ and collisional resistivity $\tilde{\eta} \simeq$ $10^{-14}$, both nonrelativistic and ultrarelativistic expressions lead to the energy on the order of $500 \mathrm{keV}$. This estimate seems to be in reasonable agreement with the RHESSI observations that imply the presence of a high-energy break in the range of 100-200 keV (Holman et al. 2003). If the resistivity is anomalously enhanced, $\tilde{\eta} \simeq 10^{-8}$, the electron energy gain $\mathcal{E}_{3 \mathrm{~d}} \simeq$ $0.5 \mathrm{GeV}$ turns out to be roughly the same as the predicted proton energy. Note that this estimate can be reduced because of synchrotron energy losses. Observationally, electron energies in excess of several tens of $\mathrm{MeV}$ are associated with the impulsive electron-rich solar flares (Rieger et al. 1998).

\section{Discussion}

The WKB method has been used in this paper to determine the acceleration times and energy gains for charged particles accelerated by the electric field that is associated with fan magnetic reconnection in the solar corona. The main result of the paper is that the energies of flare-accelerated particles can be limited by the escape time from the fan rather than by the total electric potential at the reconnection site. For typical coronal parameters, the estimates of the previous section show that the finite escape time limits proton energies if the Lundquist number is less than $10^{12}$ and electron energies if the Lundquist number is less than $10^{18}$. The same coronal parameters lead to estimates for the proton energies of the order of a few $\mathrm{MeV}$ and the electron energies of the order of a few hundred $\mathrm{keV}$ in the case of the classical electric resistivity. These estimates seem to agree with typical solar flare observations. Much higher proton and electron energies (up to a few hundred $\mathrm{MeV}$ ) can be reached if the resistivity is anomalously enhanced.

Recently Dalla \& Browning (2005) determined particle orbits near a magnetic null by integrating the equation of motion numerically and discussed particle acceleration by the reconnection electric field in the solar corona. In contrast to the work of Dalla \& Browning (2005), who focused on linear spine reconnection, in this paper the acceleration process in a nonlinear fan reconnection regime is analyzed. It is worthwhile to emphasize the key differences between the reconnection model used in this paper and the reconnection model used by Dalla \& Browning (2005).

First, spine reconnection appears to be ineffective as a flare energy release mechanism, at least based on the analysis of the available flux pile-up solutions (Craig \& Watson 2000). On the contrary, fan reconnection models lead to plausible current densities, electric fields, and flare energy release rates (Litvinenko \& Craig 2000; Craig \& Watson 2000). If the same mechanism is responsible for the overall energy release and particle acceleration in flares, then these calculations suggest that fan reconnection is a better candidate for particle acceleration.

Second, the electric and magnetic fields used by Dalla \& Browning (2005) follow from a linear ideal MHD solution for slow spine reconnection, derived by Priest \& Titov (1996). Because the solution neglects deviations of the magnetic field from the potential field, Dalla \& Browning (2005) could not describe the significant effect of the reconnecting magnetic field that acts to stabilize the particle motion near the null, whereas the potential background field causes the particle escape (see 
Sect. 3). It is an interplay between these two effects that determines the acceleration time and the resulting energy gain. The field structure adopted in this paper takes the reconnecting magnetic field into account.

A weakness of all test particle studies is that they cannot give a self-consistent description of magnetic reconnection. Fully kinetic reconnection models should be developed in order to describe the energy release and particle acceleration by collisionless reconnection. Yet test particle studies provide valuable information because full particle simulations remain limited to two-dimensional magnetic fields (Drake et al. 2005).

The particle energy gains are estimated in this paper based on the time spent by the particles near the magnetic null. The technique developed by Dalla \& Browning (2005) allows one to compute the particle trajectories near the null explicitly, which gives a much more detailed description of the acceleration process. Thus particle acceleration in solar flares can be better understood by applying the technique to acceleration in fan reconnection geometries.

Acknowledgements. Discussions with Prof. I. J. D. Craig and Dr. J. Heerikhuisen and constructive criticism by the anonymous referee are gratefully acknowledged. This work was supported by NSF grant ATM-0136718, NASA grant NNG05GM43G, and the PPARC Short Term Visitor grant to the Astronomy and Astrophysics group at the University of Glasgow.

\section{References}

Ambrosiano, J., Matthaeus, W. H., Goldstein, M. L., \& Plante, D. 1988, J. Geophys. Res., 93, 14383

Aulanier, G., DeLuca, E. E., Antiochos, S. K., McMullen, R. A., \& Golub, L. 2000, ApJ, 540, 1126
Brown, M. R., Cothran, C. D., Landreman, M., Schlossberg, D., \& Matthaeus, W. H. 2002, ApJ, 577, L63

Browning, P. K., \& Vekstein, G. E. 2001, J. Geophys. Res., 106, 18677

Bruhwiler, D. L., \& Zweibel, E. G. 1992, J. Geophys. Res., 97, 10825

Bulanov, S. V., \& Cap, F. 1988, Sov. Astron., 32, 436

Bulanov, S. V., \& Sasorov, P. V. 1976, Sov. Astron., 19, 464

Craig, I. J. D., \& Henton, S. M. 1995, ApJ, 450, 280

Craig, I. J. D., \& Fabling, R. B. 1996, ApJ, 462, 969

Craig, I. J. D., \& Litvinenko, Y. E. 2002, ApJ, 570, 387

Craig, I. J. D., \& Watson, P. G. 2000, Sol. Phys., 194, 251

Craig, I. J. D., Fabling, R. B., Henton, S. M., \& Rickard, G. J. 1995, ApJ, 455, L197

Dalla, S., \& Browning, P. K. 2005, A\&A, 436, 1103

Dmitruk, P., Matthaeus, W. H., Seenu, N., \& Brown, M. R. 2003, ApJ, 597, L81

Drake, J. F., Shay, M. A., Thongthai, W., \& Swisdak, M. 2005, Phys. Rev. Lett., 94, 095001

Efthymiopoulos, C., Gontikakis, C., \& Anastasiadis, A. 2005, A\&A, 443, 663

Fletcher, L., Metcalf, T. R., Alexander, D., Brown, D. S., \& Ryder, L. A. 2001, ApJ, 554, 451

Heerikhuisen, J., Litvinenko, Y. E., \& Craig, I. J. D. 2002, ApJ, 566, 512

Heerikhuisen, J., \& Craig, I. J. D. 2005, Sol. Phys., 222, 95

Holman, G. D., Sui, L., Schwartz, R. A., \& Emslie, A. G. 2003, ApJ, 595, L97

LaRosa, T. N. 1992, ApJ, 396, 289

Lin, R. P., Krucker, S., Hurford, G. J., et al. 2003, ApJ, 595, L69

Litvinenko, Y. E. 1996, ApJ, 462, 997

Litvinenko, Y. E. 2003, Sol. Phys., 212, 379

Litvinenko, Y. E., \& Craig, I. J. D. 2000, ApJ, 544, 1101

Martens, P. C. H. 1988, ApJ, 330, L131

Martens, P. C. H., \& Young, A. 1990, ApJS, 73, 333

Priest, E. R., \& Forbes, T. G. 2000, Magnetic Reconnection: MHD Theory and Applications (Cambridge University Press)

Priest, E. R., \& Titov, V. S. 1996, Phil. Trans. Roy. Soc. Lond. A, 354, 2951

Rieger, E., Gan, W. Q., \& Marschhäuser, H. 1998, Sol. Phys., 183, 123

Ryan, J. M. 2000, Space Sci. Rev., 93, 581

Schopper, R., Birk, G. T., \& Lesch, H. 1999, Phys. Plasmas, 6, 4318

Speiser, T. W. 1965, J. Geophys. Res., 70, 4219

Turkmani, R., Vlahos, L., Galsgaard, K., Cargill, P. J., \& Isliker, H. 2005, ApJ, 620, L59

Wood, P., \& Neukirch, T. 2005, Sol. Phys., 226, 73 